\title{
Research Article \\ Quaternionic Lattice Structures for Four-Channel Paraunitary Filter Banks
}

\author{
Marek Parfieniuk and Alexander Petrovsky \\ Department of Real-Time Systems, Faculty of Computer Science, Bialystok Technical University,
} Wiejska 45A street, 15-351 Bialystok, Poland

Received 31 December 2005; Revised 1 October 2006; Accepted 9 October 2006

Recommended by Gerald Schuller

\begin{abstract}
A novel approach to the design and implementation of four-channel paraunitary filter banks is presented. It utilizes hypercomplex number theory, which has not yet been employed in these areas. Namely, quaternion multipliers are presented as alternative paraunitary building blocks, which can be regarded as generalizations of Givens (planar) rotations. The corresponding quaternionic lattice structures maintain losslessness regardless of coefficient quantization and can be viewed as extensions of the classic twoband lattice developed by Vaidyanathan and Hoang. Moreover, the proposed approach enables a straightforward expression of the one-regularity conditions. They are stated in terms of the lattice coefficients, and thus can be easily satisfied even in finite-precision arithmetic.
\end{abstract}

Copyright (c) 2007 M. Parfieniuk and A. Petrovsky. This is an open access article distributed under the Creative Commons Attribution License, which permits unrestricted use, distribution, and reproduction in any medium, provided the original work is properly cited.

\section{INTRODUCTION}

Paraunitary filter banks (PUFBs) can be considered the most important among multirate systems [1]. This results from the fact that such filter banks are lossless in addition to guaranteeing perfect reconstruction. A clear relation between the fullband and subband signal energies greatly simplifies theoretical considerations, and hence makes PUFBs useful for applications such as image coding.

The paraunitary property means that the basis functions related to the subbands of a filter bank are orthogonal. However, it is more convenient to work with the analysis polyphase transfer matrix $\mathbf{E}(z)$, which is paraunitary if $\mathbf{E}^{H}\left(z^{-1}\right) \mathbf{E}(z)=c \mathbf{I}_{M}$, where $c$ is a nonzero constant and $M$ denotes the number of channels [2]. Thus, instead of constraining the impulse response coefficients, the usual way to obtain a PUFB is to compose its polyphase matrix from suitable building blocks. From a different point of view, the matrix is appropriately factorized. In this way, other properties of the filter frequency responses can be simultaneously imposed, such as linear phase (LP), pairwisemirror-image (PMI) symmetry, and regularity. The selection and arrangement of factorization components are decisive.
Lattice and dyadic-based factorizations of paraunitary polyphase matrices can be distinguished. The first approach utilizes Givens (planar) rotations [2]. They are implemented with the help of a specific structure, whose shape is the reason for using the name "lattice." The second technique is based on Householder reflections and degree-one building blocks, which are of a different nature [3]. The lattice structures are more frequently used because the structural imposition of the above-mentioned additional properties is easier [4-6].

A serious practical problem with the factorizations for PUFBs is that they lose essential properties in the case of finite-precision implementation. The only exception is the two-band lattice structure reported in [7]. These facts are not widely known because the effects of coefficient quantization in PUFBs were studied only in [8]. This is undoubtedly a consequence of the growing popularity of lifting factorizations, which guarantee perfect reconstruction under finite precision $[9,10]$. However, they lead to biorthogonal systems with a complicated relation between the fullband and subband signal energies.

In this paper, we propose a novel approach to the design and implementation of four-band PUFBs. It utilizes hypercomplex number theory, which has not yet been employed in these areas. Namely, quaternion multipliers are presented as 
alternative paraunitary building blocks, which can be viewed as generalizations of Givens rotations. The lattice structures based on them maintain losslessness regardless of coefficient quantization [11]. Moreover, the one-regularity conditions can be expressed in terms of the lattice coefficients and thus satisfied even under finite precision [12].

The limitation of the applicability of the technique to the case of four channels is undoubtedly a serious disadvantage. However, the proposed solution can be recognized as an extension of the two-band lossless lattice presented in [7]. Moreover, our development can stimulate further researches aimed at its generalization, on the one hand, and practical applications, on the other hand.

The organization of the paper is as follows. In Section 2, the conventional lattice structures for PUFBs are briefly reviewed to provide the necessary background for further discussion. Losslessness and regularity are approached more closely, and the effect of coefficient quantization on these properties is accentuated. Section 3 introduces a quaternionic multiplier as an alternative building block for fourchannel PUFBs. In Section 4, quaternionic variants of the factorizations from Section 2 are derived, as well as the oneregularity conditions on their coefficients. The advantages of the proposed solution, related to finite-precision implementations, are emphasized. The obtained results are exploited in Section 5, where three representative PUFB design examples are shown. Finally, some concluding remarks are given in Section 6.

Notations 1. Column vectors are denoted by lowercase boldfaced characters, whereas matrices by the uppercase ones. The notation $a_{m n}$ refers to the $(m, n)$ entry of a matrix A. $\mathbf{I}_{m}$ and $\mathbf{J}_{m}$ denote the $m \times m$ identity and reverse identity matrices, respectively. The superscript $T$ stands for transposition. Quantization is indicated with $\mathcal{Q}(\cdot)$. Three specific vectors $\mathbf{e}=\left[\begin{array}{llll}1 & 0 & 0 & 0\end{array}\right]^{T}, \mathbf{a}=\left[\begin{array}{llll}1 & 1 & 0 & 0\end{array}\right]^{T}$, and $\mathbf{o}=\left[\begin{array}{llll}1 & 1 & 1 & 1\end{array}\right]^{T}$ are helpful. The $L_{2}$-norm is considered in our discussion.

\section{CONVENTIONAL LATTICE STRUCTURES}

\subsection{Four-channel general PUFB}

The most essential issue in lossless system design is how to obtain an $M \times M$ paraunitary polyphase transfer matrix $\mathbf{E}(z)$ of a given McMillan degree [2]. No other properties are required.

At the first successful attempt to solve this problem [13], the factorization

$$
\mathbf{E}(z)=\mathbf{R}_{N-1} \hat{\Lambda}(z) \mathbf{R}_{N-2} \hat{\Lambda}(z) \cdots \mathbf{R}_{1} \widehat{\Lambda}(z) \mathbf{E}_{0}
$$

was used. It contains the delays

$$
\widehat{\Lambda}(z)=\operatorname{diag}\left(z^{-1}, \mathbf{I}_{M-1}\right)
$$

and orthogonal matrices: a general one, $\mathbf{E}_{0}$, with $M(M-1) / 2$ degrees of freedom, and $\mathbf{R}_{i}, i=1, \ldots, N-1$, constrained to have $M-1$ of these. Both kinds of matrices are commonly implemented using Givens (planar) rotations, each of which corresponds to one degree of freedom [2].

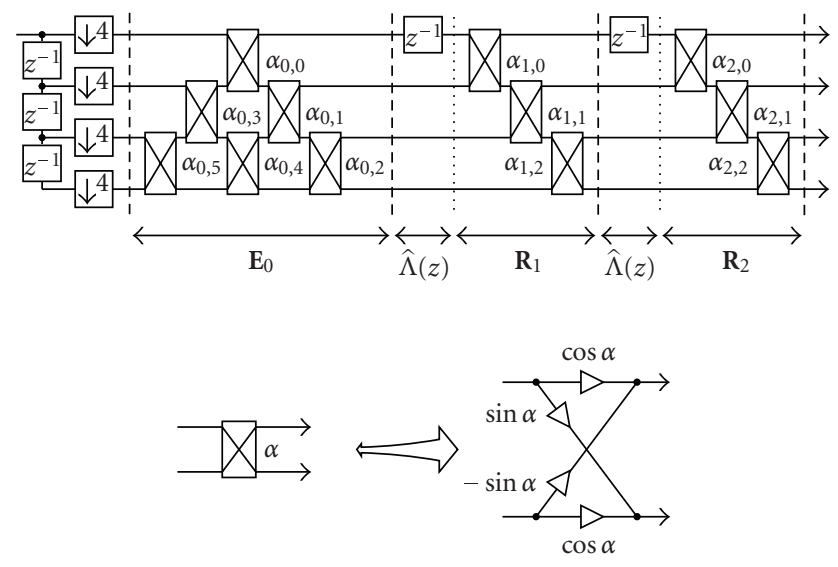

FIgURE 1: Conventional plane rotation-based lattice structure for 4-channel general PUFB $(N=3)$.

For $M=4$ and $N=3$, the details of this approach, which is tightly connected with the QR decomposition of a matrix, are explained in the scheme shown in Figure 1.

\subsection{Four-channel LP PUFB}

Linear phase responses of a filter bank are necessary to use symmetric extension to handle the boundaries of finitelength signals [14]. Therefore, LP PUFBs are very important from a practical point of view, especially in image processing. For these systems, the best known factorization of the polyphase transfer matrix assumes $M$ to be an even number and has the following form $[4,15]$ :

$$
\mathbf{E}(z)=\mathbf{G}_{N-1}(z) \mathbf{G}_{N-2}(z) \cdots \mathbf{G}_{1}(z) \mathbf{E}_{0},
$$

in which

$$
\begin{gathered}
\mathbf{E}_{0}=\frac{1}{\sqrt{2}} \boldsymbol{\Phi}_{0} \mathbf{W} \operatorname{diag}\left(\mathbf{I}_{M / 2}, \mathbf{J}_{M / 2}\right), \\
\mathbf{G}_{i}(z)=\frac{1}{2} \boldsymbol{\Phi}_{i} \mathbf{W} \boldsymbol{\Lambda}(z) \mathbf{W}, \quad i=1, \ldots, N-1,
\end{gathered}
$$

where

$$
\begin{gathered}
\mathbf{W}=\left[\begin{array}{cc}
\mathbf{I}_{M / 2} & \mathbf{I}_{M / 2} \\
\mathbf{I}_{M / 2} & -\mathbf{I}_{M / 2}
\end{array}\right], \\
\boldsymbol{\Lambda}(z)=\operatorname{diag}\left(\mathbf{I}_{M / 2}, z^{-1} \mathbf{I}_{M / 2}\right), \\
\boldsymbol{\Phi}_{i}=\operatorname{diag}\left(\mathbf{U}_{i}, \mathbf{V}_{i}\right) .
\end{gathered}
$$

The design freedom is related to the $M / 2 \times M / 2$ orthogonal matrices $\mathbf{U}_{i}$ and $\mathbf{V}_{i}$, which are again parameterized using Givens rotations. For $M=4$ and $N=3$, this approach leads to the structure shown in Figure 2. It should be noted that a $2 \times 2$ orthogonal matrix corresponds to a single rotation.

A relatively recent result is the simplification of the above factorization derived in [16]. Namely, for $i>0, \mathbf{U}_{i}$ can be replaced with the identity matrix, so that

$$
\boldsymbol{\Phi}_{i}=\operatorname{diag}\left(\mathbf{I}_{M / 2}, \mathbf{V}_{i}\right), \quad i>0,
$$

without affecting the completeness of the factorization. 


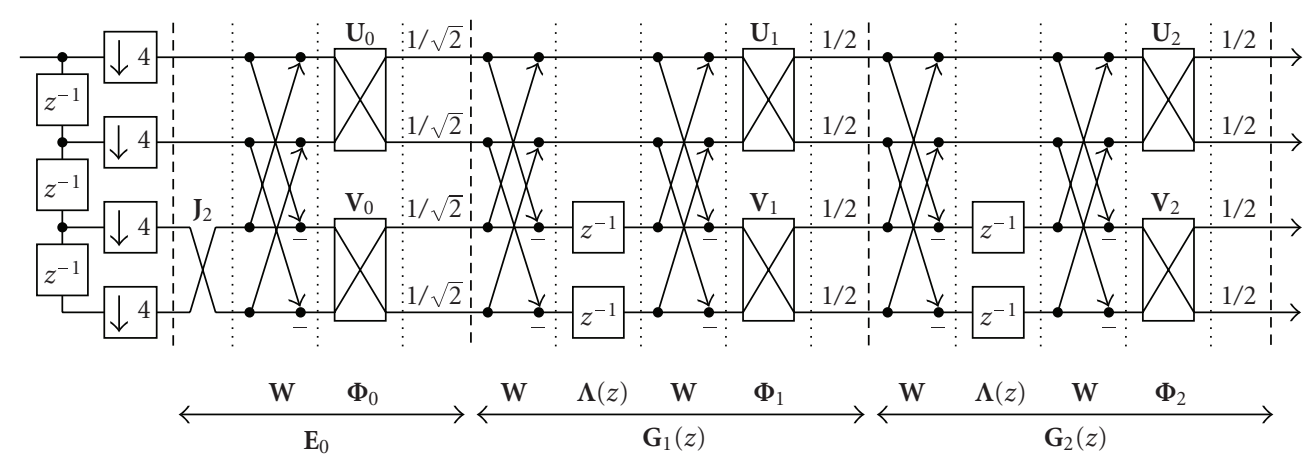

FIGURE 2: Conventional lattice structure for 4-channel LP PUFB $(N=3)$.

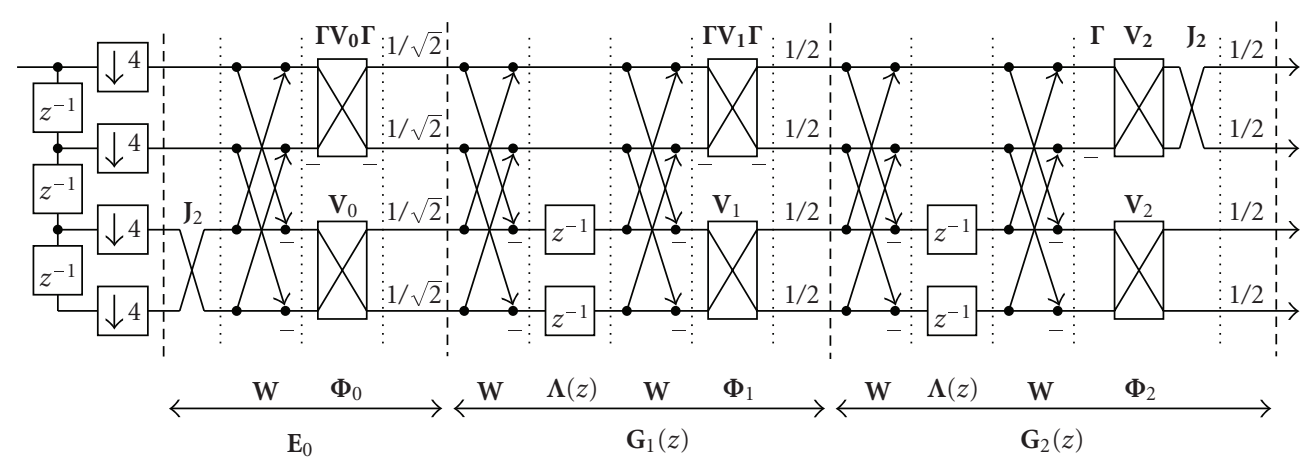

FIGURE 3: Conventional lattice structure for 4-channel PMI LP PUFB $(N=3)$.

\subsection{Four-channel PMI LP PUFB}

Among LP PUFBs, there are systems with pairwise-mirrorimage symmetric frequency responses [17]. This property means that the magnitude responses of the pairs of filters are symmetric with respect to $\pi / 2$, which can be expressed in terms of the transfer functions or impulse responses of the analysis filters as

$$
H_{M-1-k}(z)= \pm H_{k}(-z)
$$

or

$$
h_{M-1-k}(n)= \pm(-1)^{n} h_{k}(n),
$$

respectively, where $k=0, \ldots, N-1$ and $n=0, \ldots, L-1$, assuming that the filters are of length $L$.

In the case of an even $M$, PMI symmetry can be easily obtained by slightly modifying the lattice factorization for LP PUFBs. Namely, it is sufficient to associate $\mathbf{U}_{i}$ with $\mathbf{V}_{i}$ in (8) so that

$$
\begin{gathered}
\mathbf{U}_{i}=\mathbf{\Gamma V}_{i} \boldsymbol{\Gamma}, \quad i=0, \ldots, N-2, \\
\mathbf{U}_{N-1}=\mathbf{J}_{M / 2} \mathbf{V}_{N-1} \boldsymbol{\Gamma},
\end{gathered}
$$

where $\boldsymbol{\Gamma}$ is the diagonal matrix whose diagonal entries are $\gamma_{m m}=(-1)^{m-1}, m=1, \ldots, M / 2$.
As the number of the degrees of design freedom is reduced, the optimization of filter bank coefficients is easier, which was the main motivation behind the development of such systems. Recently, it has been shown how to achieve further simplifications [18].

For $M=4$ and $N=3$, such an approach leads to the structure shown in Figure 3.

\subsection{Construction of synthesis filter bank}

To process a signal in subbands, both analysis and synthesis filter banks are needed. In practice, the synthesis computational scheme is constructed by arranging the inverses of the components of the factorization of the analysis polyphase transfer matrix in reverse order. It is noteworthy, however, that in the paraunitary case, the synthesis filters are simply the time-reversed version of the analysis ones.

\subsection{Coefficient quantization effects}

\subsubsection{Losslessness}

All presented conventional factorizations lose paraunitary property in the case of coefficient quantization. Even perfect reconstruction is not provided by finite-precision lattice structures. This is because a quantized Givens rotation 
matrix, for example,

$$
\left[\begin{array}{cccc}
1 & 0 & 0 & 0 \\
0 & 1 & 0 & 0 \\
0 & 0 & \mathcal{Q}(\cos \alpha) & -\mathcal{Q}(\sin \alpha) \\
0 & 0 & \mathcal{Q}(\sin \alpha) & \mathcal{Q}(\cos \alpha)
\end{array}\right]
$$

is not orthogonal as there are two different column norms: 1 and $\sqrt{\mathcal{Q}^{2}(\cos \alpha)+Q^{2}(\sin \alpha)} \neq 1$, and only one nonorthogonal component is enough to destroy the losslessness of an entire factorization [8].

\subsubsection{Regularity}

Coefficient quantization also affects the regularity of a filter bank. This property is crucial for low bit-rate coding where subband coefficients are aggressively quantized, as it alleviates blocking artifacts [14]. The concept originates from wavelet theory, where it is a property of scaling functions and wavelets, critical for smooth signal approximation $[19,20]$. However, it is not straightforward to extend the notion to discrete-time systems, especially to $M$-band ones in which $M>2$.

For an $M$-band filter bank, regularity can be defined as the number of zeros at the mirror (aliasing) frequencies $2 k \pi / M, k=1, \ldots, M-1$, of the lowpass filter $H_{0}(z)$. To obtain $K$ degrees of regularity, the polyphase matrix $\mathbf{E}(z)$ must satisfy the condition [6]

$$
\left.\frac{d^{n}}{d z^{n}}\left\{\mathbf{E}\left(z^{M}\right)\left[\begin{array}{llll}
1 & z^{-1} & \cdots & z^{-(M-1)}
\end{array}\right]^{T}\right\}\right|_{z=1}=c_{n} \mathbf{e}
$$

with $c_{n} \neq 0$ for $n=0, \ldots, K-1$. In particular, for the oneregularity $(K=1)$ and four bands $(M=4)$, the above expression simplifies to

$$
\mathbf{E}(1) \mathbf{o}=c_{0} \mathbf{e}
$$

It is easy to verify that this is equivalent to have zero magnitude responses of all bandpass filters $H_{k}(z), k=1, \ldots, M-1$, at DC (zero) frequency. Thus a constant input is entirely captured by the lowpass filter, and there is no leakage to the remaining bands, which would cause the checkerboard artifact in the case of an image coding application [14].

Conventionally, the regularity conditions are expressed in terms of the angles of the Givens rotations which form a lattice structure $[6,14]$. However, such an approach is of limited practical importance, as quantization of rotation matrices changes the corresponding angles, which destroys regularity. So it is more advantageous to have the regularity conditions expressed directly in terms of lattice coefficients.

\section{QUATERNIONS AND ORTHOGONAL MATRICES}

\subsection{Quaternions}

Quaternions were discovered by Hamilton [21]. They are hypercomplex numbers of the form [22]

$$
q=q_{1}+q_{2} i+q_{3} j+q_{4} k, \quad q_{1}, q_{2}, q_{3}, q_{4} \in \mathbb{R},
$$

with one real and three distinct imaginary parts. The imaginary units $i, j$, and $k$ are related by the following equations:

$$
\begin{gathered}
i^{2}=j^{2}=k^{2}=i j k=-1, \\
i j=-j i=k, \quad j k=-k j=i, \quad k i=-i k=j .
\end{gathered}
$$

They define quaternion multiplication so that

$$
\begin{aligned}
p q= & \left(p_{1} q_{1}-p_{2} q_{2}-p_{3} q_{3}-p_{4} q_{4}\right) \\
& +\left(p_{1} q_{2}+p_{2} q_{1}+p_{3} q_{4}-p_{4} q_{3}\right) i \\
& +\left(p_{1} q_{3}+p_{3} q_{1}+p_{4} q_{2}-p_{2} q_{4}\right) j \\
& +\left(p_{1} q_{4}+p_{4} q_{1}+p_{2} q_{3}-p_{3} q_{2}\right) k
\end{aligned}
$$

which is associative and distributive, but noncommutative $(p q \neq q p)$ unless one of the operands is a scalar. This mainly distinguishes quaternions, as the definitions of other operations are nothing more than simple extensions of those related to complex numbers. As examples, we can consider the addition

$$
p \pm q=p_{1} \pm q_{1}+\left(p_{2} \pm q_{2}\right) i+\left(p_{3} \pm q_{3}\right) j+\left(p_{4} \pm q_{4}\right) k
$$

the conjugate

$$
\bar{q}=q_{1}-q_{2} i-q_{3} j-q_{4} k
$$

and the norm (modulus)

$$
|q|=\sqrt{q \bar{q}}=\sqrt{\bar{q} q}=\sqrt{q_{1}^{2}+q_{2}^{2}+q_{3}^{2}+q_{4}^{2}} .
$$

The division is defined as the multiplication by the reciprocal

$$
q^{-1}=\frac{\bar{q}}{|q|^{2}}
$$

which satisfies the identity

$$
q q^{-1}=q^{-1} q=1
$$

The modulus $|q|$ forms the basis for the polar representation [21]

$$
\begin{gathered}
q_{1}=|q| \cos \phi, \\
q_{2}=|q| \sin \phi \cos \psi, \\
q_{3}=|q| \sin \phi \sin \psi \cos \chi, \\
q_{4}=|q| \sin \phi \sin \psi \sin \chi,
\end{gathered}
$$

where the angles $0 \leq \phi \leq \pi, 0 \leq \psi \leq \pi$, and $0 \leq \chi<2 \pi$ are the three remaining degrees of freedom. Polar representation allows us to easily parameterize fixed-modulus quaternions. In our case, unit quaternions $(|q|=1)$ are of great importance. 


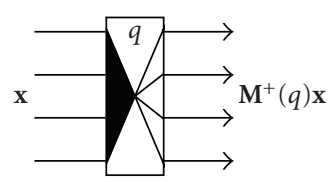

(a)

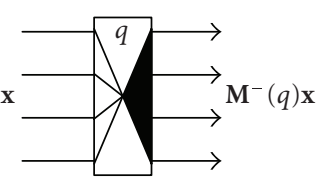

(b)
Figure 4: Graphical symbols for the quaternion multipliers whose coefficient $q$ is (a) the left multiplication operand and (b) the right multiplication operand, respectively.

\subsection{Quaternion multiplication matrices}

Because quaternions can be identified with four-element column vectors:

$$
q \Leftrightarrow \mathbf{q}=\left[\begin{array}{llll}
q_{1} & q_{2} & q_{3} & q_{4}
\end{array}\right]^{T},
$$

all operations on hypercomplex numbers can be consistently represented in vector-matrix notation. We are particularly interested in multiplication, which can be written in two equivalent forms as

$$
\begin{aligned}
p q & \Leftrightarrow \underbrace{\left[\begin{array}{cccc}
p_{1} & -p_{2} & -p_{3} & -p_{4} \\
p_{2} & p_{1} & -p_{4} & p_{3} \\
p_{3} & p_{4} & p_{1} & -p_{2} \\
p_{4} & -p_{3} & p_{2} & p_{1}
\end{array}\right]}_{\mathbf{M}^{+}(p)} \times\left[\begin{array}{l}
q_{1} \\
q_{2} \\
q_{3} \\
q_{4}
\end{array}\right] \\
& =\underbrace{\left[\begin{array}{cccc}
q_{1} & -q_{2} & -q_{3} & -q_{4} \\
q_{2} & q_{1} & q_{4} & -q_{3} \\
q_{3} & -q_{4} & q_{1} & q_{2} \\
q_{4} & q_{3} & -q_{2} & q_{1}
\end{array}\right]}_{\mathbf{M}^{-}(q)} \times\left[\begin{array}{l}
p_{1} \\
p_{2} \\
p_{3} \\
p_{4}
\end{array}\right] .
\end{aligned}
$$

Thus two different multiplication matrices exist, the left$\mathbf{M}^{+}(\cdot)$ and right-operand $\mathbf{M}^{-}(\cdot)$ one.

In the following discussion, we restrict ourselves to unit quaternion multiplication matrices. To represent quaternion multipliers graphically, we also introduce the symbols shown in Figure 4.

Both matrices are orthogonal as

$$
\mathbf{M}^{ \pm}(q)^{-1}=\mathbf{M}^{ \pm}(q)^{T},
$$

and have determinant +1 . Hence, they belong to the $4 \times 4$ special orthogonal group commonly referred to as $\mathrm{SO}(4)$ [23]. They also form groups with respect to multiplication, which implies the following identities:

$$
\begin{aligned}
\mathbf{M}^{+}\left(q_{N-1}\right) \cdots \mathbf{M}^{+}\left(q_{0}\right) & =\mathbf{M}^{+}\left(q_{N-1} \cdots q_{0}\right), \\
\mathbf{M}^{-}\left(q_{N-1}\right) \cdots \mathbf{M}^{-}\left(q_{0}\right) & =\mathbf{M}^{-}\left(q_{0} \cdots q_{N-1}\right) .
\end{aligned}
$$

Another interesting and useful relation is

$$
\mathbf{M}^{ \pm}(q)^{T}=\mathbf{M}^{ \pm}(\bar{q}) .
$$

From a PUFB perspective, the connections between quaternion multiplication matrices and arbitrary $4 \times 4$ orthogonal ones are intriguing. To make the paper comprehensive, we have decided to repeat the derivations from $[24,25]$

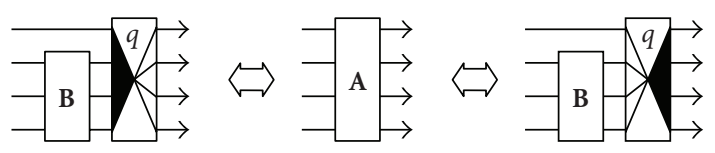

FIGURE 5: Structural transformation corresponding to (31).

in a quite large extent, but the emphasis is placed on slightly different nuances.

\subsection{Reduction of a $4 \times 4$ orthogonal matrix}

Theorem 1. Every $4 \times 4$ orthogonal matrix $\mathbf{A}$ can be represented as the product

$$
\mathbf{A}=\mathbf{M}^{ \pm}(a) \operatorname{diag}(1, \mathbf{B}),
$$

where

$$
a=a_{11}-a_{21} i-a_{31} j-a_{41} k
$$

and $\mathbf{B}$ is a $3 \times 3$ orthogonal matrix.

Proof. As $\mathbf{A}$ and $\mathbf{M}^{ \pm}(a)$ are both orthogonal, $\mathbf{M}^{ \pm}(a)^{T} \mathbf{A}$ must be orthogonal as well. The quaternion $a$ is constructed so as to have the inner product of the first columns of $\mathbf{A}$ and $\mathbf{M}^{ \pm}(a)$ equal to unity. This is the value of the $(1,1)$ st element of $\mathbf{M}^{ \pm}(a)^{T} \mathbf{A}$, so all the remaining elements in the first row and column of this matrix must be zeros. Thus the rest of its elements forms a $3 \times 3$ orthogonal matrix $\mathbf{B}$.

The corresponding structural transformation is shown in Figure 5. It should be noted that the reducing ability of unit quaternion multiplication matrices suggests their tight connections with Givens rotations, which are commonly used in matrix parameterization via $\mathrm{QR}$ decomposition, as it has been mentioned earlier. One quaternion multiplication is related to three degrees of freedom and can be treated as a fourdimensional generalization of a Givens rotation [11].

\subsection{Parameterization of $a 4 \times 4$ orthogonal matrix}

Theorem 2 (see [24]). For every orthogonal $4 \times 4$ matrix A, there exists a unique (up to signs) pair of unit quaternions $p$ and $q$ such that

$$
\mathbf{A}=\mathbf{M}^{+}(p) \mathbf{M}^{-}(q)=\mathbf{M}^{-}(q) \mathbf{M}^{+}(p) .
$$

Proof. We begin by decomposing the given matrix $\mathbf{A}$ according to $(31)$ and then deal with $\operatorname{diag}(1, \mathbf{B})$. It is known [25] that the latter matrix can be represented using one unit quaternion $b$ as

$$
\mathbf{M}^{+}(b) \mathbf{M}^{-}(\bar{b})=\operatorname{diag}(1, \mathbf{B}),
$$

where

$$
\mathbf{B}=\left[\begin{array}{ccc}
b_{1}^{2}+b_{2}^{2}-b_{3}^{2}-b_{4}^{2} & 2\left(-b_{1} b_{4}+b_{2} b_{3}\right) & 2\left(b_{1} b_{3}+b_{2} b_{4}\right) \\
2\left(b_{1} b_{4}+b_{3} b_{2}\right) & b_{1}^{2}-b_{2}^{2}+b_{3}^{2}-b_{4}^{2} & 2\left(-b_{1} b_{2}+b_{3} b_{4}\right) \\
2\left(-b_{1} b_{3}+b_{4} b_{2}\right) & 2\left(b_{1} b_{2}+b_{4} b_{3}\right) & b_{1}^{2}-b_{2}^{2}-b_{3}^{2}+b_{4}^{2}
\end{array}\right] \text {. }
$$


The equations

$$
\begin{gathered}
b_{1}^{2}=\frac{1}{4}\left(1+b_{11}+b_{22}+b_{33}\right), \\
b_{2}^{2}=\frac{1}{4}\left(1+b_{11}-b_{22}-b_{33}\right), \\
b_{3}^{2}=\frac{1}{4}\left(1-b_{11}+b_{22}-b_{33}\right), \\
b_{4}^{2}=\frac{1}{4}\left(1-b_{11}-b_{22}+b_{33}\right), \\
b_{1} b_{2}=\frac{1}{4}\left(b_{32}-b_{23}\right), \quad b_{1} b_{3}=\frac{1}{4}\left(b_{13}-b_{31}\right), \\
b_{1} b_{4}=\frac{1}{4}\left(b_{21}-b_{12}\right), \quad b_{2} b_{3}=\frac{1}{4}\left(b_{12}+b_{21}\right), \\
b_{2} b_{4}=\frac{1}{4}\left(b_{13}+b_{31}\right), \quad b_{3} b_{4}=\frac{1}{4}\left(b_{23}+b_{32}\right),
\end{gathered}
$$

which can be easily derived, allow us to calculate $b$ from $\mathbf{B}$.

This system of equations is overdetermined as the number of equations exceeds the number of unknowns. To avoid a contradiction, the equation which gives the $b_{k}$ of a maximum absolute value should be selected from among (36). Then it must be supplemented by the three equations in (37) which involve $b_{k}$, to allow us to determine all components of the quaternion $b$. It should be noted that the squares at the left-hand side of (36) make $-b$ an equivalent solution. Finally, we get the desired factorization

$$
\mathbf{A}=\mathbf{M}^{+}(a) \mathbf{M}^{+}(b) \mathbf{M}^{-}(\bar{b})=\mathbf{M}^{+}(a b) \mathbf{M}^{-}(\bar{b})
$$

based on the quaternions $p=a b$ and $q=\bar{b}$.

It should be emphasized that the matrix product (33) is commutative, though the product of the related quaternions is not. The theorem is also true after the transition to $-p$ and $-q$.

\subsection{Quaternion multiplier as paraunitary building block}

The parameterization (33) has several advantages which make quaternion multipliers interesting paraunitary building blocks.

First of all, a quantized quaternion multiplication matrix, for example,

$$
\left[\begin{array}{cccc}
\mathcal{Q}\left(q_{1}\right) & -\mathcal{Q}\left(q_{2}\right) & -\mathcal{Q}\left(q_{3}\right) & -\mathcal{Q}\left(q_{4}\right) \\
\mathcal{Q}\left(q_{2}\right) & \mathcal{Q}\left(q_{1}\right) & -\mathcal{Q}\left(q_{4}\right) & \mathcal{Q}\left(q_{3}\right) \\
\mathcal{Q}\left(q_{3}\right) & \mathcal{Q}\left(q_{4}\right) & \mathcal{Q}\left(q_{1}\right) & -\mathcal{Q}\left(q_{2}\right) \\
\mathcal{Q}\left(q_{4}\right) & -\mathcal{Q}\left(q_{3}\right) & \mathcal{Q}\left(q_{2}\right) & \mathcal{Q}\left(q_{1}\right)
\end{array}\right]
$$

still has the same sets of absolute values in all its rows and columns. So the column norm is constant and is equal to $\sqrt{\mathcal{Q}\left(q_{1}\right)^{2}+\mathcal{Q}\left(q_{2}\right)^{2}+\mathcal{Q}\left(q_{3}\right)^{2}+\mathcal{Q}\left(q_{4}\right)^{2}}$, and hence the product (33) always represents an orthogonal transformation.

Moreover, it is sufficient to hold only 8 real numbers (2 quaternions) in memory, whereas the direct representation of the corresponding matrix would require to store all its 16 entries.

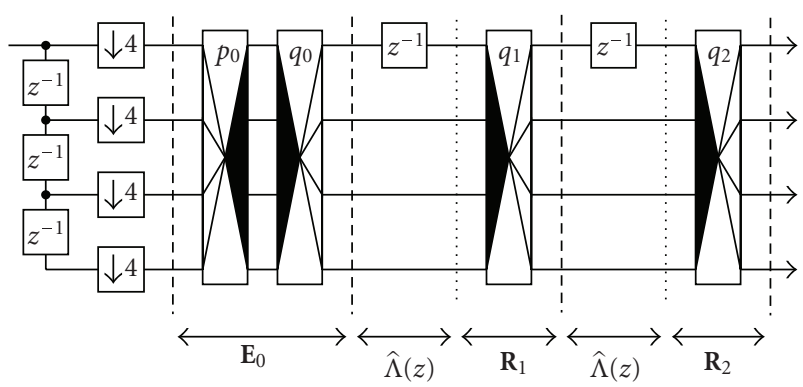

FIGURE 6: Quaternionic lattice structure for 4-channel general $\operatorname{PUFB}(N=3)$.

The specific structures of quaternion multiplication matrices allow us to perform this operation in 8 real multiplications, but the algorithm is quite intricate [26].

The possibility of multiplierless implementations is much more important. They can be realized with distributed arithmetic or using four-dimensional CORDIC algorithm. The feasibility of computation parallelization or pipelining together with the regularity of the layout of a digital circuit make quaternionic multiplier very attractive for FPGA and VLSI technologies [27].

\section{QUATERNIONIC LATTICE STRUCTURES}

\subsection{Four-channel general PUFB}

Theorem 3 (see [11]). The quaternionic variant of the factorization (1) for a 4-channel general PUFB results from the following substitution:

$$
\begin{gathered}
\mathbf{E}_{0}^{\prime}=\mathbf{M}^{+}\left(q_{0}\right) \mathbf{M}^{-}\left(p_{0}\right), \\
\mathbf{R}_{i}^{\prime}=\mathbf{M}^{ \pm}\left(q_{i}\right), \quad i=1, \ldots, N-1,
\end{gathered}
$$

where $p_{0}$ and all $q_{i}$ are unit-norm quaternions.

Proof. Both of the theorems from the previous section, which concern $4 \times 4$ orthogonal matrices, are exploited. According to (31), the matrices $\mathbf{R}_{i}$ in (1) can be represented as

$$
\mathbf{R}_{i}=\mathbf{M}^{ \pm}\left(q_{i}\right) \operatorname{diag}\left(1, \mathbf{B}_{i}\right)
$$

Since

$$
\operatorname{diag}\left(1, \mathbf{B}_{i}\right) \hat{\Lambda}(z)=\widehat{\Lambda}(z) \operatorname{diag}\left(1, \mathbf{B}_{i}\right)
$$

the $3 \times 3$ orthogonal matrix $\mathbf{B}_{i}$ can be moved to the preceding stage and multiplied by $\mathbf{R}_{i-1}$. The same procedure can be applied to the resulting orthogonal matrix. Starting from $\mathbf{R}_{N-1}$, we process the subsequent stages to reach $\mathbf{E}_{0}$ and to apply (33).

Figure 6 shows the corresponding quaternionic lattice structure. 


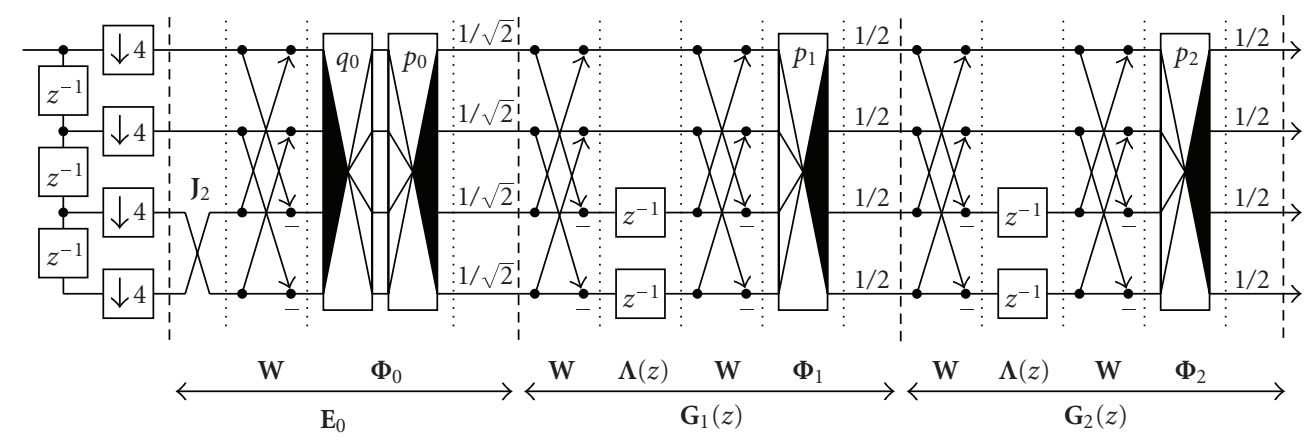

FIgURE 7: Quaternionic lattice structure for 4-channel LP PUFB $(N=3)$.

Theorem 4 (see [12]). A four-band general PUFB determined by (1) in conjunction with (40) and (41) is one-regular if and only if

$$
p_{0}= \pm \frac{1}{2} \bar{o} \overline{q_{N-1} \cdots q_{0}}
$$

under the assumption that the left-operand multiplication matrix is used in (41).

Proof. By substituting $\mathrm{E}(z)$ with (1) in (16), and then using (40) and (41), we get

$$
\mathbf{M}^{+}\left(q_{N-1}\right) \cdots \mathbf{M}^{+}\left(q_{0}\right) \mathbf{M}^{-}\left(p_{0}\right) \mathbf{o}=c_{0} \mathbf{e}
$$

as $\hat{\boldsymbol{\Lambda}}(1)=\mathbf{I}_{4}$. A simple analysis of the norms of the factors in this expression gives the value of $c_{0}$. It must be \pm 2 as the norm of $\mathbf{o}$ equals 2 , and those of $\mathbf{e}$ and the rows/columns of the quaternion matrices are unity. Hence, by exploiting (29a) too, we can write

$$
\mathbf{M}^{+}\left(q_{N-1} \cdots q_{0}\right) \mathbf{M}^{-}\left(p_{0}\right) \mathbf{o}= \pm 2 \mathbf{e} .
$$

This clearly suggests to make $p_{0}$ constrained, so we use (30) to obtain

$$
\mathbf{M}^{-}\left(p_{0}\right) \mathbf{o}= \pm 2 \mathbf{M}^{+}\left(\overline{q_{N-1} \cdots q_{0}}\right) \mathbf{e} .
$$

This matrix-vector expression can be interpreted as the quaternionic equation

$$
o p_{0}= \pm 2 \overline{q_{N-1} \cdots q_{0}}
$$

in which $p_{0}$ is unknown. The left multiplication by $\bar{o} / 4$ leads to the solution, or (44).

The above result can be easily adapted to the case of the right-operand multiplication matrix in (41). We omit this for brevity reasons.

\subsection{Four-channel LP PUFB}

Theorem 5 (see [28]). The conventional, presented in Section 2.2, factorization for 4-channel LP PUFBs changes into a quaternionic alternative when

$$
\begin{gathered}
\boldsymbol{\Phi}_{0}^{\prime}=\mathbf{M}^{-}\left(p_{0}\right) \mathbf{M}^{+}\left(q_{0}\right), \\
\boldsymbol{\Phi}_{i}^{\prime}=\mathbf{M}^{-}\left(p_{i}\right), \quad i=1, \ldots, N-1,
\end{gathered}
$$

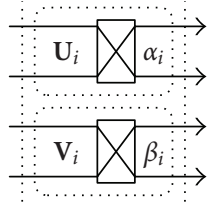

(a)

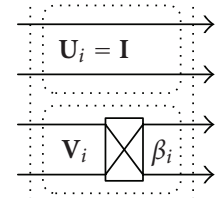

(b)

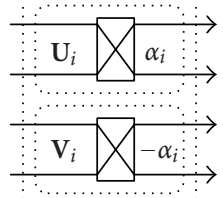

(c)
Figure 8: (a) Conventional, (b) simplified, and (c) quaternionic realizations of $\boldsymbol{\Phi}_{i}$.

are used instead of (8). All $p_{i}$ and $q_{0}$ are unit quaternions which have the two last imaginary parts (related to $j$ and $k$ ) zeroed, so they are constrained to be complex numbers in fact.

Proof. Theorem 2 allows us to decompose each matrix $\boldsymbol{\Phi}_{i}$ defined by (8) in the following way:

$$
\boldsymbol{\Phi}_{i}=\mathbf{M}^{-}\left(p_{i}\right) \mathbf{M}^{+}\left(q_{i}\right) .
$$

The block-diagonal structure of $\boldsymbol{\Phi}_{i}$ is inherited by the quaternion multiplication matrices and this is the cause of the degeneration of the corresponding hypercomplex numbers to the complex ones. It is easy to check that

$$
\begin{aligned}
\mathbf{M}^{+}\left(q_{i}\right) \mathbf{W} & =\mathbf{W M}^{+}\left(q_{i}\right), \\
\mathbf{M}^{+}\left(q_{i}\right) \boldsymbol{\Lambda}(z) & =\boldsymbol{\Lambda}(z) \mathbf{M}^{+}\left(q_{i}\right) .
\end{aligned}
$$

Thus $\mathbf{M}^{+}\left(q_{i}\right)$ can be moved to the preceding stage $\mathbf{G}_{i-1}(z)$, which leads to (50). As the product $\boldsymbol{\Phi}_{i-1} \mathbf{M}^{+}\left(q_{i}\right)$ maintains orthogonality and a block-diagonal structure, the procedure can be repeated on it. The only exception is at $\boldsymbol{\Phi}_{0}$, which must be represented using both quaternion multiplication matrices.

The corresponding lattice structure is shown in Figure 7. It is noteworthy that by quaternionic factorization, the number of different coefficients is reduced with respect to the conventional approach [4] and the same as in the simplified variant derived in [16]. However, the computational complexity remains unchanged. The differences between the mentioned realizations of $\boldsymbol{\Phi}_{i}$ are explained in Figure 8. 


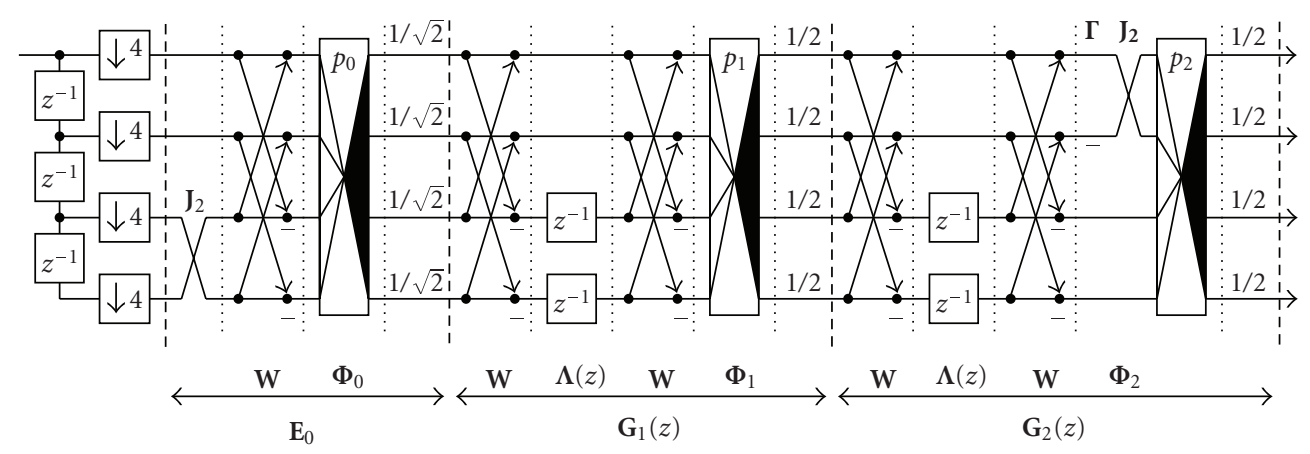

FIGURE 9: Quaternionic lattice structure for 4-channel PMI LP PUFB $(N=3)$.

Theorem 6 (see [12]). A 4-band LP PUFB realized using the quaternionic approach is one-regular if and only if

$$
q_{0}= \pm \frac{1}{\sqrt{2}} \overline{p_{0} \cdots p_{N-1}} \bar{a}
$$

Proof. As in the case of a general PUFB, the first step is to expand (16) in accordance with the considered factorization of $\mathbf{E}(z)$. We get

$$
\mathbf{M}^{-}\left(p_{N-1}\right) \cdots \mathbf{M}^{-}\left(p_{0}\right) \mathbf{M}^{+}\left(q_{0}\right) \sqrt{2} \mathbf{a}=c_{0} \mathbf{e}
$$

as $\mathbf{W} \boldsymbol{\Lambda}(1) \mathbf{W}=2 \mathbf{I}_{4}$ and $\mathbf{W} \operatorname{diag}\left(\mathbf{I}_{2}, \mathbf{J}_{2}\right) \mathbf{o}=\mathbf{a}$. The value of $c_{0}$ again results from the examination of the norms of the factors and must be \pm 2 as the norm of a equals $\sqrt{2}$, while the remaining ones are unity. Applying (29b), we obtain

$$
\mathbf{M}^{-}\left(p_{0} \cdots p_{N-1}\right) \mathbf{M}^{+}\left(q_{0}\right) \mathbf{a}= \pm \sqrt{2} \mathbf{e}
$$

and see that it is the easiest to make $q_{0}$ dependent on the remaining coefficients. The identity (30) allows us to write the matrix equation

$$
\mathbf{M}^{+}\left(q_{0}\right) \mathbf{a}= \pm \sqrt{2} \mathbf{M}^{-}\left(\overline{p_{0} \cdots p_{N-1}}\right) \mathbf{e}
$$

and then convert it into the quaternionic equivalent

$$
q_{0} a= \pm \sqrt{2} \overline{p_{0} \cdots p_{N-1}}
$$

The right multiplication by $\bar{a} / 2$ gives the desired regularity constraint (54) on $q_{0}$.

\subsection{Four-channel PMI LP PUFB}

Theorem 7 (see [28]). The constraints (12)-(13) on the matrices used in the factorization from Section 2.3, for 4-channel PMI LP PUFBs, can be satisfied by taking

$$
\begin{aligned}
& \boldsymbol{\Phi}_{i}^{\prime}=\mathbf{M}^{-}\left(p_{i}\right), \quad i=0, \ldots, N-2, \\
& \boldsymbol{\Phi}_{N-1}^{\prime}=\mathbf{M}^{-}\left(p_{N-1}\right) \operatorname{diag}\left(\mathbf{J}_{2} \boldsymbol{\Gamma}, \mathbf{I}_{2}\right),
\end{aligned}
$$

where $\Gamma=\operatorname{diag}(1,-1)$ and the quaternionic coefficients $p_{i}$ are restricted to be unit complex numbers.
Proof. In the case of 4 channels, $\boldsymbol{\Gamma} \mathbf{V}_{i} \boldsymbol{\Gamma}=\mathbf{V}_{i}^{T}$, and so the first condition (12) necessary to obtain PMI symmetry directly imposes the form of $\boldsymbol{\Phi}_{i}$ which coincides with a quaternion multiplication matrix, because

$$
\boldsymbol{\Phi}_{i}=\operatorname{diag}\left(\boldsymbol{\Gamma} \mathbf{V}_{i} \boldsymbol{\Gamma}, \mathbf{V}_{i}\right)=\underbrace{\operatorname{diag}\left(\mathbf{V}_{i}^{T}, \mathbf{V}_{i}\right)}_{\mathbf{M}^{-}\left(p_{i}\right)}
$$

if $p_{i}$ is constrained to be a complex number.

The obvious identities $\mathbf{J}=\mathbf{I}$ and $\mathbf{J}_{2} \mathbf{V}_{i} \mathbf{J}_{2}=\mathbf{V}_{i}^{T}$ allow a quaternion multiplication matrix to be extracted also from $\boldsymbol{\Phi}_{N-1}$ determined by the condition (13). Namely,

$$
\begin{aligned}
\boldsymbol{\Phi}_{N-1} & =\operatorname{diag}\left(\mathbf{J}_{2} \mathbf{V}_{N-1} \boldsymbol{\Gamma}, \mathbf{V}_{N-1}\right) \\
& =\underbrace{\operatorname{diag}\left(\mathbf{V}_{N-1}^{T}, \mathbf{V}_{N-1}\right)}_{\mathbf{M}^{-}\left(p_{N-1}\right)} \operatorname{diag}\left(\mathbf{J}_{2} \boldsymbol{\Gamma}, \mathbf{I}_{2}\right) .
\end{aligned}
$$

The corresponding structure is shown in Figure 9. In the case of a PMI LP PUFB, by quaternionic factorization the number of coefficients is decreased with respect to the conventional solution and is the same as in its simplification derived in [18].

Theorem 8 (see [12]). A four-band PMI LP PUFB realized according to Theorem 7 is one-regular if and only if

$$
p_{N-1}= \pm \frac{1}{\sqrt{2}} a \overline{p_{0} \cdots p_{N-2}}
$$

Proof. Given the quaternionic factorization, we can expand (16) into

$$
\begin{aligned}
& \mathbf{M}^{-}\left(p_{N-1}\right) \operatorname{diag}\left(\mathbf{J}_{2} \boldsymbol{\Gamma}, \mathbf{I}_{2}\right) \\
& \quad \cdot \mathbf{M}^{-}\left(p_{N-2}\right) \cdots \mathbf{M}^{-}\left(p_{0}\right) \sqrt{2} \mathbf{a}=c_{0} \mathbf{e} .
\end{aligned}
$$

The value of $c_{0}$ results from norm inspection and equals \pm 2 . Noticing that

$$
\operatorname{diag}\left(\mathbf{J}_{2} \boldsymbol{\Gamma}, \mathbf{I}_{2}\right)=\frac{1}{2} \mathbf{M}^{+}(\bar{a}) \mathbf{M}^{-}(\bar{a}),
$$

and utilizing (29b) and (30), we can rewrite (64) as

$$
\frac{1}{2} \mathbf{M}^{+}(\bar{a}) \mathbf{M}^{-}(\bar{a}) \mathbf{M}^{-}\left(p_{0} \cdots p_{N-2}\right) \mathbf{a}= \pm \sqrt{2} \mathbf{M}^{-}\left(\bar{p}_{N-1}\right) \mathbf{e} .
$$


Then, the transition to quaternions yields

$$
\frac{1}{\sqrt{2} 2} \bar{a} a p_{0} \cdots p_{N-2} \bar{a}= \pm \bar{p}_{N-1},
$$

and we obtain (63) by conjugating both sides, as $\bar{a} a=2$.

\subsection{Robustness to coefficient quantization}

All of the developed lattice structures are lossless regardless of coefficient quantization. This is because the derived factorizations contain no components which become nonorthogonal when represented with finite precision. Thus, the frequency responses of such systems are always powercomplementary [2]:

$$
\sum_{k=0}^{M-1}\left|H_{k}\left(e^{j \omega}\right)\right|^{2}=c^{2}, \quad \forall \omega,
$$

though $c$ can deviate from 1 . If a compensation of this effect is desired, $c$ can be calculated as

$$
c^{2}=\left.\sum_{k=0}^{M-1}\left|H_{k}\left(e^{j \omega}\right)\right|^{2}\right|_{\omega=0}=\sum_{k=0}^{M-1}\left|\sum_{n=0}^{L-1} h_{k}(n)\right|^{2} .
$$

and the multiplication by its reciprocal can be easily embedded into the computational scheme.

The plot thickens if regularity is considered because the quaternion conditioned by the others under (44), (54), or (63) must be represented accurately. Fortunately, the necessary wordlength is finite and strictly determined by those of the remaining coefficients. Moreover, any scaling of the coefficient value does not disturb regularity.

To demonstrate that regularity can indeed be easily imposed on quaternionic lattice structures, even under finite precision, the next section shows three design examples. The obtained filter banks with rational quaternionic coefficients can be implemented using fixed-point arithmetic, possibly in multiplierless manner as in [29].

\section{DESIGN EXAMPLES}

\subsection{Coefficient synthesis methodology}

The goal was to obtain frequency-selective filter banks with high coding gains. So, the weighted sum of two criteria was used as an objective function for optimization.

The first criterion is the stopband attenuation measured in terms of energy as

$$
\varepsilon_{\mathrm{SBE}}=\sum_{k=0}^{M-1} \int_{\omega \in \Omega_{k}}\left|H_{k}\left(e^{j \omega}\right)\right|^{2} d \omega,
$$

where $\Omega_{k}$ denotes the stopband of the $k$ th filter and the number of channels, $M$, equals 4 in our case.

The second performance criterion is a coding gain defined as

$$
C G=10 \log _{10} \frac{(1 / M) \sum_{k=0}^{M-1} \sigma_{x_{k}}^{2}}{\left(\prod_{k=0}^{M-1} \sigma_{x_{k}}^{2}\right)^{1 / M}}
$$

TABLE 1: Rational coefficient values for general PUFB.

\begin{tabular}{lccccc}
\hline Coeff. & $\operatorname{Re}$ & $\operatorname{Im}_{i}$ & $\operatorname{Im}_{j}$ & $\operatorname{Im}_{k}$ & Wordlength \\
\hline$p_{0}$ & $-45 / 128$ & $9 / 16$ & $-31 / 128$ & $-5 / 8$ & 8 \\
$q_{0}$ & $-11 / 16$ & $-1 / 2$ & $1 / 16$ & $7 / 16$ & 5 \\
$q_{1}$ & $3 / 8$ & $1 / 8$ & $3 / 4$ & $-1 / 2$ & 4 \\
\hline
\end{tabular}

where $\sigma_{x_{k}}^{2}$ are the subband variances. They correspond to the diagonal elements of the autocorrelation matrix of the transformed signal, $\mathbf{R}_{y y}$ :

$$
\sigma_{x_{k}}^{2}=\left[\mathbf{R}_{y y}\right]_{k k}
$$

It can be determined as the product

$$
\mathbf{R}_{y y}=\mathbf{H R}_{x x} \mathbf{H}^{T}
$$

of the autocorrelation matrix of the input signal, $\mathbf{R}_{x x}$, and the transform matrix $\mathbf{H}$ formed from the impulse responses of the filter bank as follows:

$$
[\mathbf{H}]_{k n}=h_{k}(L-1-n),
$$

where $k=0, \ldots, M-1$ and $n=0, \ldots, L-1$.

In our experiments, the matrix $\mathbf{R}_{x x}$ was generated for an $\mathrm{AR}(1)$ input process with unit variance and the correlation coefficient of 0.95 . Such a model is particularly appropriate only for natural images, and therefore other applications will require different approaches.

In the synthesis procedures, the quaternion lattice coefficients assumed to be unconstrained in (54) and (63) were represented in the polar form (25). So the standard Matlab routines intended for an unconstrained optimization, that is, fminunc and fminsearch, could be used to search for the angles that minimize the objective function. Given infiniteprecision coefficients, we carefully converted them into rationals. This was done intuitively by hand, but the development of an advanced algorithm, like that proposed in [29], is planned.

\subsection{Design example 1: 8-tap general PUFB}

A 4-channel 8-tap PUFB was designed using the results from Section 4.1. The synthesized coefficients of quaternionic lattice structure are given in Table 1 and the corresponding magnitude responses are shown in Figure 10(a). The filter bank is characterized by a coding gain of $8.1227 \mathrm{~dB}$ and a minimum stopband attenuation of $20 \mathrm{~dB}$, so it can compete with the similar system demonstrated in [10].

The plots allow us to verify that the designed PUFB is indeed one-regular. It can be easily noticed in Figure 10(a) that only the lowpass filter has a nonzero magnitude response at DC frequency. On the other hand, the zero-pole plot in Figure 10(b) shows that the lowpass filter has a single zero in each of that points of the unit circle, which correspond 


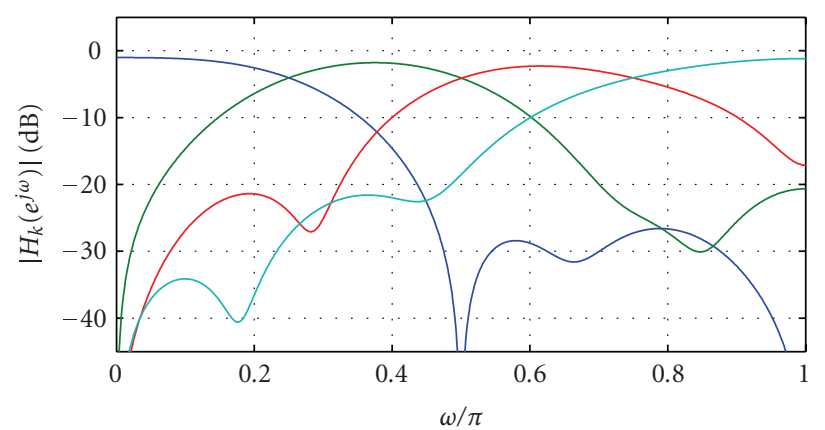

(a)

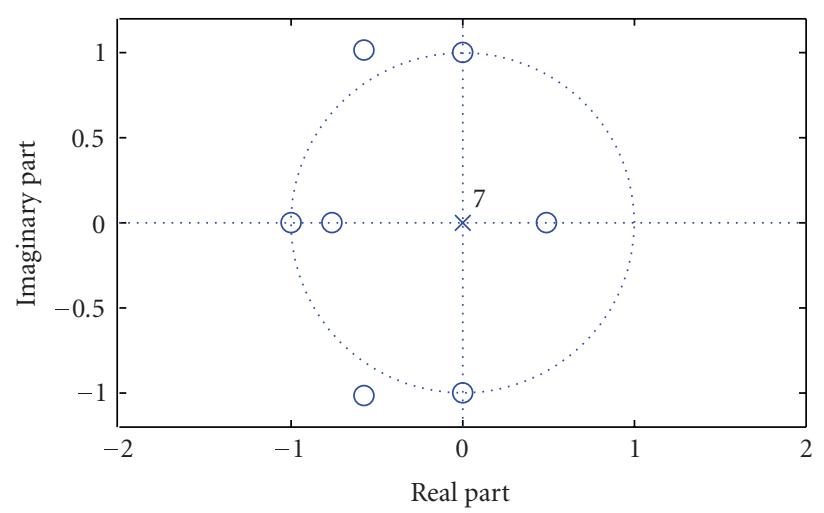

(b)
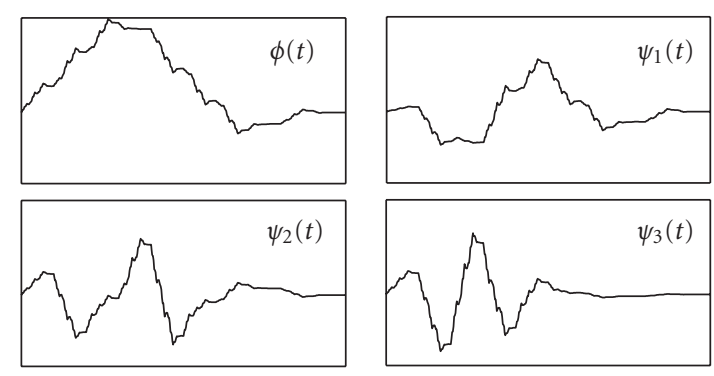

(c)

FIgURE 10: Design example of general PUFB: (a) magnitude responses, (b) zeros of $H_{0}(z)$, and (c) the scaling function and wavelets.

to the mirror aliasing frequencies. Figure 10(c) demonstrates the wavelet basis related to the system.

\subsection{Design example 2: 12-tap LP PUFB}

The second design example demonstrates the usefulness of the theory developed in Section 4.2. The hypercomplex coefficient values given in Table 2 determine the 12-tap LP PUFB which has a coding gain of $8.1845 \mathrm{~dB}$. The plots in Figure 11 allow us to evaluate the magnitude responses of the system and verify its one-regularity. The filters have good frequency selectivity. For the lowpass and highpass ones, the sidelobes are below the $-35 \mathrm{~dB}$ level, whereas for the bandpass filters, the peak amplitude of the sidelobes is about $-20 \mathrm{~dB}$.
TABLE 2: Rational coefficient values for LP PUFB.

\begin{tabular}{lccccc}
\hline Coeff. & $\mathrm{Re}$ & $\mathrm{Im}_{i}$ & $\mathrm{Im}_{j}$ & $\operatorname{Im}_{k}$ & Wordlength \\
\hline$q_{0}$ & $-231 / 512$ & $459 / 1024$ & 0 & 0 & 11 \\
$p_{0}$ & $-7 / 8$ & $-3 / 8$ & 0 & 0 & 4 \\
$p_{1}$ & $-3 / 16$ & $15 / 16$ & 0 & 0 & 5 \\
$p_{2}$ & $-9 / 16$ & $-13 / 16$ & 0 & 0 & 5 \\
\hline
\end{tabular}

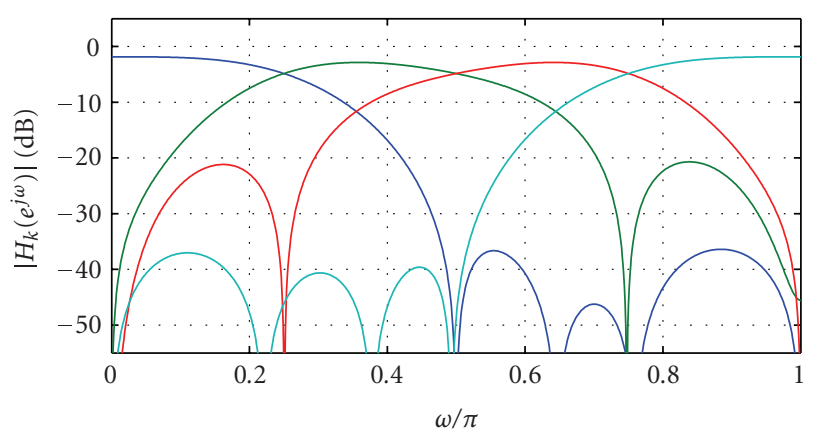

(a)

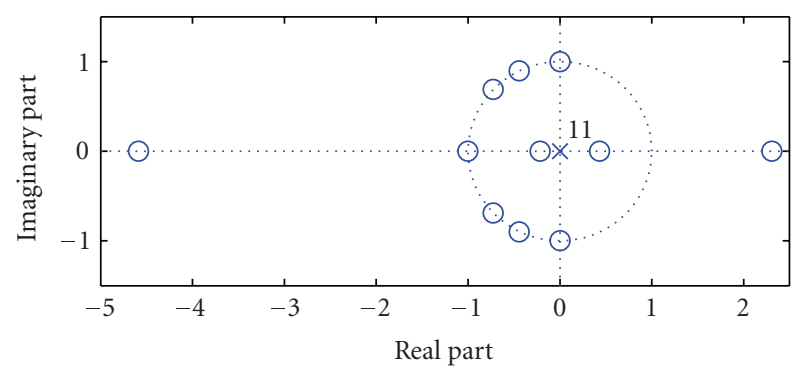

(b)
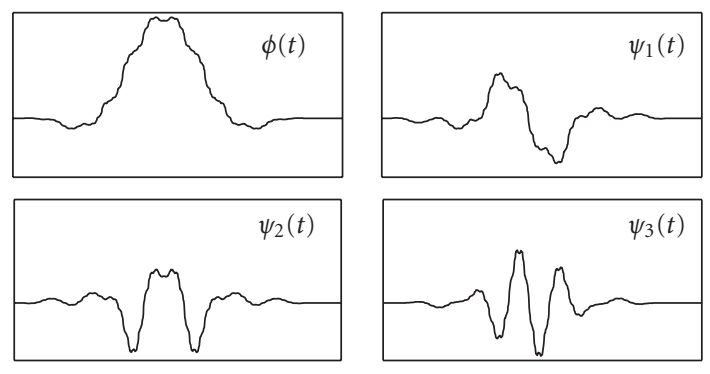

(c)

FIGURE 11: Design example of LP PUFB: (a) magnitude responses, (b) zeros of $H_{0}(z)$, and (c) the scaling function and wavelets.

\subsection{Design example 3: 12-tap PMI LP PUFB}

The results from Section 4.3 allowed us to design the 12-tap LP PUFB whose pairwise-mirror-image symmetric magnitude responses are shown in Figure 12(a). The coefficients of the quaternionic lattice are given in Table 3 . In spite of decreased design freedom and shorter coefficient wordlengths, the characteristics of the system are very close to those of the LP filter bank presented above. Namely, the coding gain is of $8.1699 \mathrm{~dB}$ and the levels of the sidelobes are of -31 and 
TABLE 3: Rational coefficient values for PMI LP PUFB.

\begin{tabular}{lccccc}
\hline Coeff. & $\operatorname{Re}$ & $\operatorname{Im}_{i}$ & $\operatorname{Im}_{j}$ & $\operatorname{Im}_{k}$ & Wordlength \\
\hline$p_{0}$ & $7 / 8$ & $3 / 8$ & 0 & 0 & 4 \\
$p_{1}$ & $3 / 16$ & -1 & 0 & 0 & 5 \\
$p_{2}$ & $-17 / 128$ & $43 / 64$ & 0 & 0 & 8 \\
\hline
\end{tabular}

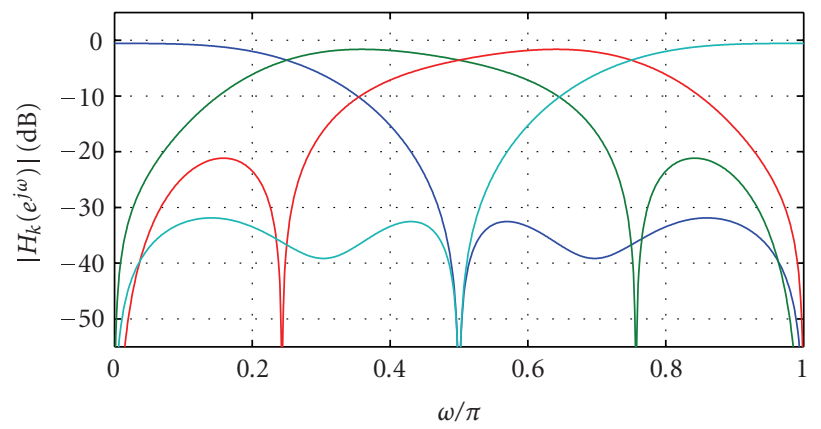

(a)

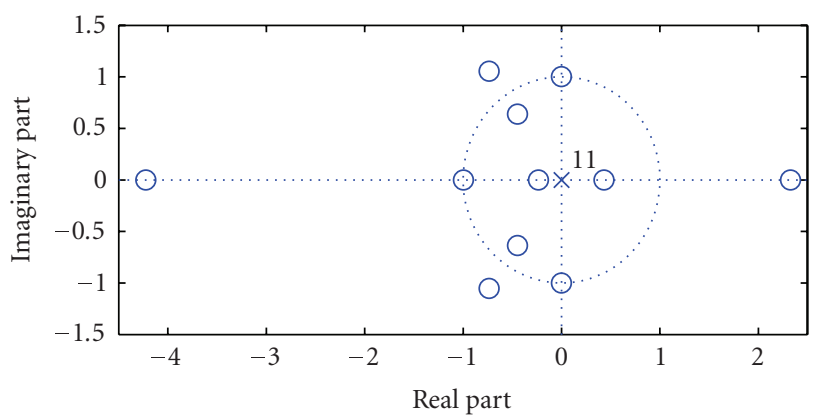

(b)
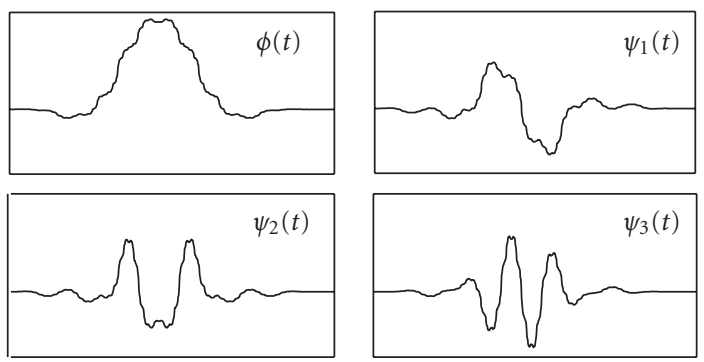

(c)

Figure 12: Design example of PMI LP PUFB: (a) magnitude responses, (b) zeros of $H_{0}(z)$, and (c) the scaling function and wavelets.

$-20 \mathrm{~dB}$. The reason for this is the similarity of the zero locations shown in Figure 12(b) to those in Figure 11(b). The differences between the wavelet bases are almost unnoticeable.

\section{CONCLUSION}

The developed quaternionic approach to the design and implementation of four-band PUFBs seems to be very competitive with the conventional techniques. Its unique advantage is the structural imposition of paraunitary property (losslessness) even with finite-precision arithmetic. It also enables the straightforward expression of the one-regularity conditions in terms of the coefficients of the quaternionic lattice structure, which is also advantageous in fixed-point implementations. So the solution is especially interesting from a practical point of view.

\section{ACKNOWLEDGMENTS}

This work was supported by the Polish Ministry of Science and Higher Education (MNiSzW) in years 2005-2006 (Grant no. 3 T11F 014 29). It was also partially supported by Bialystok Technical University under the Grant W/WI/2/05.

\section{REFERENCES}

[1] P. P. Vaidyanathan and Z. Doǧanata, "The Role of lossless systems in modern digital signal processing: a tutorial," IEEE Transactions on Education, vol. 32, no. 3, pp. 181-197, 1989.

[2] P. P. Vaidyanathan, Multirate Systems and Filter Banks, Prentice-Hall, Englewood Cliffs, NJ, USA, 1993.

[3] P. P. Vaidyanathan, T. Q. Nguyen, Z. Doğanata, and T. Saramaki, "Improved technique for design of perfect reconstruction FIR QMF banks with lossless polyphase matrices," IEEE Transactions on Acoustics, Speech, and Signal Processing, vol. 37, no. 7, pp. 1042-1056, 1989.

[4] A. K. Soman, P. P. Vaidyanathan, and T. Q. Nguyen, "Linear phase paraunitary filter banks: theory, factorizations and designs," IEEE Transactions on Signal Processing, vol. 41, no. 12, pp. 3480-3496, 1993.

[5] A. K. Soman and P. P. Vaidyanathan, "A complete factorization of paraunitary matrices with pairwise mirror-image symmetry in the frequency domain," IEEE Transactions on Signal Processing, vol. 43, no. 4, pp. 1002-1004, 1995.

[6] S. Oraintara, T. D. Tran, P. N. Heller, and T. Q. Nguyen, "Lattice structure for regular paraunitary linear-phase filterbanks and M-band orthogonal symmetric wavelets," IEEE Transactions on Signal Processing, vol. 49, no. 11, pp. 2659-2672, 2001.

[7] P. P. Vaidyanathan and P.-Q. Hoang, "Lattice structures for optimal design and robust implementation of two-band perfect reconstruction QMF banks," IEEE Transactions on Acoustic, Speech, and Signal Processing, vol. 36, no. 1, pp. 81-94, 1988.

[8] P. P. Vaidyanathan, "On coefficient-quantization and computational roundoff effects in lossless multirate filter banks," IEEE Transactions on Signal Processing, vol. 39, no. 4, pp. 1006$1008,1991$.

[9] Y.-J. Chen and K. S. Amaratunga, " $M$-channel lifting factorization of perfect reconstruction filter banks and reversible $M$ band wavelet transforms," IEEE Transactions on Circuits and Systems II: Analog and Digital Signal Processing, vol. 50, no. 12, pp. 963-976, 2003.

[10] Y.-J. Chen, S. Oraintara, and K. S. Amaratunga, "Dyadicbased factorizations for regular paraunitary filterbanks and $M$-band orthogonal wavelets with structural vanishing moments," IEEE Transactions on Signal Processing, vol. 53, no. 1, pp. 193-207, 2005.

[11] M. Parfieniuk and A. Petrovsky, "Quaternionic building block for paraunitary filter banks," in Proceedings of the 12th European Signal Processing Conference (EUSIPCO '04), pp. 12371240, Vienna, Austria, September 2004. 
[12] M. Parfieniuk and A. Petrovsky, "Quaternionic formulation of the first regularity for four-band paraunitary filter banks," in Proceedings of IEEE International Symposium on Circuits and Systems (ISCAS '06), pp. 883-886, Kos, Greece, May 2006.

[13] Z. Doğanata, P. P. Vaidyanathan, and T. Q. Nguyen, "General synthesis procedures for FIR lossless transfer matrices, for perfect-reconstruction multirate filter bank applications," IEEE Transactions on Acoustics, Speech, and Signal Processing, vol. 36, no. 10, pp. 1561-1574, 1988.

[14] G. Strang and T. Q. Nguyen, Wavelets and Filter Banks, Wellesley-Cambridge Press, Wellesley, Mass, USA, 1996.

[15] R. L. de Queiroz, T. Q. Nguyen, and K. R. Rao, “The GenLOT: generalized linear-phase lapped orthogonal transform," IEEE Transactions on Signal Processing, vol. 44, no. 3, pp. 497-507, 1996.

[16] L. Gan and K.-K. Ma, "A simplified lattice factorization for linear-phase perfect reconstruction filter bank," IEEE Signal Processing Letters, vol. 8, no. 7, pp. 207-209, 2001.

[17] T. Q. Nguyen and P. P. Vaidyanathan, "Maximally decimated perfect-reconstruction FIR filter banks with pairwise mirrorimage analysis (and synthesis) frequency responses," IEEE Transactions on Acoustics, Speech, and Signal Processing, vol. 36, no. 5, pp. 693-706, 1988.

[18] L. Gan and K.-K. Ma, "A simplified lattice factorization for linear-phase paraunitary filter banks with pairwise mirror image frequency responses," IEEE Transactions on Circuits and Systems II: Express Briefs, vol. 51, no. 1, pp. 3-7, 2004.

[19] O. Rioul, "Regular wavelets: a discrete-time approach," IEEE Transactions on Signal Processing, vol. 41, no. 12, pp. 35723579, 1993.

[20] P. Steffen, P. N. Heller, R. A. Gopinath, and C. S. Burrus, "Theory of regular $M$-band wavelet bases," IEEE Transactions on Signal Processing, vol. 41, no. 12, pp. 3497-3511, 1993.

[21] W. R. Hamilton, "On quaternions; or on a new system of imaginaries in algebra," The London, Edinburgh and Dublin Philosophical Magazine and Journal of Science, vol. 25, pp. 489-495, 1844.

[22] I. L. Kantor and A. S. Solodovnikov, Hypercomplex Numbers: An Elementary Introduction to Algebras, Springer, New York, NY, USA, 1989.

[23] A. Baker, Matrix Groups: An Introduction to Lie Group Theory, Springer, London, UK, 2002.

[24] H. G. Baker, "Quaternions and orthogonal 4x4 real matrices," Tech. Rep., June 1996, http://www.gamedev.net/reference/ articles/article428.asp.

[25] E. Salamin, "Application of quaternions to computation with rotations," Tech. Rep., Stanford AI Lab, Stanford, Calif, USA, 1979.

[26] T. D. Howell and J. C. Lafon, "The complexity of the quaternion product," Tech. Rep. TR 75-245, Cornell University, Ithaca, NY, USA, June 1975, http://citeseer.ist.psu.edu/ howell75complexity.html.

[27] M. Parfieniuk and A. Petrovsky, "Implementation perspectives of quaternionic component for paraunitary filter banks," in Proceedings of the International Workshop on Spectral Methods and Multirate Signal Processing (SMMSP '04), pp. 151-158, Vienna, Austria, September 2004.

[28] M. Parfieniuk and A. Petrovsky, "Linear phase paraunitary filter banks based on quaternionic component," in Proceedings of International Conference on Signals and Electronic Systems (ICSES '04), pp. 203-206, Poznań, Poland, September 2004.
[29] Y.-J. Chen, S. Oraintara, T. D. Tran, K. Amaratunga, and T. Q. Nguyen, "Multiplierless approximation of transforms with adder constraint," IEEE Signal Processing Letters, vol. 9, no. 11, pp. 344-347, 2002.

Marek Parfieniuk was born in Bialystok, Poland, in 1975. He received his M.S. degree in computer science, with honors, from $\mathrm{Bi}$ alystok Technical University, in 2000. Currently, he is completing the procedures for the Ph.D. degree. Since 2000, he has been an Assistant Lecturer at Faculty of Computer Science, Bialystok Technical University. From 2000 to 2003, he also worked for ComputerLand S.A. as an Enterprise Software Developer.

Alexander Petrovsky received the Dipl.Ing. degree in computer engineering, in 1975, and the Ph.D. degree, in 1980, both from the Minsk Radio-Engineering Institute, Minsk, Belarus. In 1989, he received the Doctor of Science degree from The Institute of Simulation Problems in Power Engineering, Academy of Science, Kiev, Ukraine. In 1975, he joined Minsk RadioEngineering Institute. He became a Re-

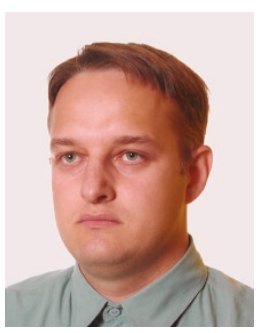
search Worker and Assistant Professor; and since 1980, he has been an Associate Professor at the Computer Science Department. From 1983 to 1984, he was a Research Worker at the Royal Holloway College and the Imperial College of Science and Technology, University of London, London, UK. Since May 1990, he has been a Professor and Head of the Computer Engineering Department, the Belarusian State University of Informatics and Radioelectronics, and he is with the Real-Time Systems department, Faculty of Computer Science, Bialystok Technical University, Poland. Recently, his main research interests are acoustic signal processing, such as speech and audio codings, noise reduction and acoustic echo cancellation, robust speech recognition, and real-time signal processing. He is a Member of Russian A. S. Popov Society for Radioengineering, Electronics, and Communications, and an Editorial Staff Member of the Russian journal Digital Signal Processing, AES, IEEE, EURASIP. 